\title{
Data Cost Games as an Application of 1-Concavity in Cooperative Game Theory
}

\author{
Dongshuang Hou ${ }^{1,2}$ and Theo Driessen ${ }^{2}$ \\ ${ }^{1}$ Department of Applied Mathematics, Northwestern Polytechnical University, Xi'an 710129, China \\ ${ }^{2}$ Department of Applied Mathematics, Faculty of Electrical Engineering, Mathematics and Computer Science, \\ University of Twente, 7500 AE Enschede, The Netherlands
}

Correspondence should be addressed to Dongshuang Hou; dshhou@126.com

Received 29 November 2013; Accepted 17 January 2014; Published 11 March 2014

Academic Editor: Pu-yan Nie

Copyright (c) 2014 D. Hou and T. Driessen. This is an open access article distributed under the Creative Commons Attribution License, which permits unrestricted use, distribution, and reproduction in any medium, provided the original work is properly cited.

The main goal is to reveal the 1-concavity property for a subclass of cost games called data cost games. The motivation for the study of the 1-concavity property is the appealing theoretical results for both the core and the nucleolus, in particular their geometrical characterization as well as their additivity property. The characteristic cost function of the original data cost game assigns to every coalition the additive cost of reproducing the data the coalition does not own. The underlying data and cost sharing situation is composed of three components, namely, the player set, the collection of data sets for individuals, and the additive cost function on the whole data set. The proof of 1-concavity is direct, but robust to a suitable generalization of the characteristic cost function. As an adjunct, the 1-concavity property is shown for the subclass of so-called "bicycle" cost games, inclusive of the data cost games in which the individual data sets are nested in a decreasing order.

\section{The Data Sharing Situation and the Data Cost Game}

This paper broadens the game theoretic approach to the data sharing situation initiated by Dehez and Tellone [1]. The origin of their mathematical study is the data and cost sharing problem faced by the European chemical industry. Following the regulation imposed by the European Commission under the acronym "REACH" (Registration, Evaluation, Authorization and restriction of Chemical substances), manufacturers and importers are required to collect safety information on the properties of their chemical substances. There are about 30,000 substances and an average of 100 parameters for each substance. Chemical firms are required to register the information in a central database run by the European Chemicals Agency (ECHA). By 2018, this regulation program REACH requires submission of a detailed analysis of the chemical substances produced or imported. Chemical firms are encouraged to cooperate by sharing the data they have collected over the past. To implement this data sharing problem, a compensation mechanism is needed.
This data sharing problem can be specified as follows. A finite group of firms agrees to undertake a joint venture that requires the combination of various complementary inputs held by some of them. These inputs are nonrival but excludable goods, that is, public goods with exclusion such as knowledge, data or information, and patents or copyrights (the consumption of which by individuals can be controlled, measured, and subjected to payment or other contractual limitations). In what follows we use the common term data to cover generically these goods. Each firm owns a subset of data. No a priori restrictions are imposed on the individual data sets. In addition, with each type of data, there is a replacement cost corresponding to it, for example, the present cost of duplicating the data (or the cost of developing alternative technologies). Because these public goods are already available, their costs are sunk. In summary, the data sharing situation involves a finite group of agents and data sets owned by individual agents, as well as a discrete list of costs of data.

In the setting of cooperative attitudes by chemical firms, the main question arises how to compensate the firms for the data they contribute to share. The design of a compensation 
mechanism, however, is fully equivalent to the selection among existing solution concepts in the mathematical field called cooperative game theory. In fact, the solution part of cooperative game theory aims at solving any allocation problem by proposing rules based on certain fairness properties. For that purpose, the data and cost sharing situation needs to be interpreted as a mathematical model called a cooperative game by specifying its fundamental characteristic cost function. We adopt Dehez and Tellone's game theoretic model in which the cost associated to any nonempty group of agents is simply the sum of costs of the missing data, that is, the total cost of data the group does not own. In this framework, no costs are charged to the whole group of agents. The socalled data cost games are therefore compensation games to which standard cost allocation rules can be applied, such as the Shapley value $[2,3]$, the nucleolus [4], and the core. The determination of these game theoretic solution concepts may be strongly simplified whenever the underlying characteristic cost function satisfies, by chance, one or another appealing property. The main purpose of this paper is to establish the so-called 1-concavity property for the class of data cost games, which has not yet been revealed. The impact of the 1-concavity property is fundamental for the uniform determination of both solution concepts the core and the nucleolus [5].

Definition 1 (see [1] with adapted notation). (i) A data and cost sharing situation is given by the 3 -tuple $\mathscr{D} \mathscr{C}=(N, \mathscr{D}, \mathscr{C})$, where $N$ is the finite set of agents, $\mathscr{D}=\left(D_{i}\right)_{i \in N}$ a collection of sets $D_{i} \subseteq D, i \in N$, of data, and $\mathscr{C}=\left(c_{j}\right)_{j \in D}$ a collection of costs of data. So, $D=\bigcup_{i \in N} D_{i}$ denotes the whole data set.

(ii) Given the set $N$ of agents, let $\mathscr{P}(N)=\{S \mid S \subseteq N\}$ denote the power set of $N$. For every coalition $S \subseteq N, S \neq \emptyset$, let $D_{S}=\bigcup_{i \in S} D_{i}$ denote the data set of $S$. For every subset $A \subseteq D$ of data, let $c(A)=\sum_{j \in A} c_{j}$ denote its additive cost, whereas $c(\emptyset)=0$.

(iii) With every data and cost sharing situation $\mathscr{D} \mathscr{C}=$ $(N, \mathscr{D}, \mathscr{C})$, there is the associated data cost game $\left\langle N, C_{\mathscr{D} \mathscr{C}}\right\rangle$, of which the characteristic cost function $C_{\mathscr{D} \mathscr{C}}: \mathscr{P}(N) \rightarrow \mathbb{R}$ is given by $C_{\mathscr{D} \mathscr{C}}(\emptyset)=0$ and for all $S \subseteq N, S \neq \emptyset$,

$$
C_{\mathscr{D C}}(S)=\sum_{j \in D \backslash D_{S}} c_{j}
$$

Shortly, $C_{\mathscr{D} \mathscr{C}}(S)=c\left(D \backslash D_{S}\right)=c(D)-c\left(D_{S}\right)$.

By (1), the so-called data cost $C_{\mathscr{D} \mathscr{C}}(S)$ of coalition $S$ equals the additive cost of duplicating the missing data, that is, costs of data the coalition does not own. Without loss of generality, it is tacitly supposed that there exist no overall missing data; that is, $D=D_{N}$; otherwise the data cost of every nonempty coalition $S$ would increase with the same cost amounting $c\left(D \backslash D_{N}\right)=c(D)-c\left(D_{N}\right)$. In our framework, no data costs are charged to the whole set of agents; that is, $C_{\mathscr{D C}}(N)=0$. Obviously, every data cost game $\left\langle N, C_{\mathscr{D C}}\right\rangle$ satisfies both the (decreasing) monotonicity (i.e., $C_{\mathscr{D C}}(S) \geq C_{\mathscr{D} \mathscr{C}}(T)$ for all $S \subseteq$ $T \subseteq N, S \neq \emptyset$, due to $D_{S} \subseteq D_{T}$ ) and subadditivity as well (i.e., $C_{\mathscr{D} \mathscr{C}}(S \cup T) \leq C_{\mathscr{D} \mathscr{C}}(S)+C_{\mathscr{D C}}(T)$ for all $S, T \subseteq N$ with $S \cap$ $T=\emptyset)$.
Definition 2 (see [5-7]). A cooperative cost game $\langle N, C\rangle$ with player set $N$ is said to satisfy the 1-concavity property if its characteristic cost function $C: \mathscr{P}(N) \rightarrow \mathbb{R}$ satisfies

$$
\begin{gathered}
C(N) \leq C(S)+\sum_{i \in N \backslash S} \Delta_{i}(N, C) \quad \forall S \subseteq N, S \neq N, S \neq \emptyset, \\
C(N) \geq \sum_{i \in N} \Delta_{i}(N, C)
\end{gathered}
$$

where $\Delta_{i}(N, C)=C(N)-C(N \backslash\{i\}) \quad \forall i \in N$.

Condition (2) requires that the cost $C(N)$ of the formation of the grand coalition $N$ can be covered by any coalitional cost $C(S)$ together with the marginal costs $\Delta_{i}(N, C), i \in N \backslash S$, of all the complementary players. According to condition (3), all these marginal costs are weakly insufficient to cover the overall cost $C(N)$. In the framework of data cost games, the latter condition (3) holds trivially due to the compensation assumption $C_{\mathscr{D} \mathscr{C}}(N)=0$.

For 1-concave or convex games $(N, v)$, its core and nucleolus have very nice structures, respectively. Its core is the convex hull of the extreme points, which are given by $\vec{b}^{v}-$ $g^{v}(N) \cdot \vec{e}_{i}, i \in N$, where $b_{i}^{v}=v(N)-v(N \backslash\{i\})$ and $g^{v}(N)=$ $b^{v}(N)-v(N)$, while its nucleolus agrees with the center of gravity of the core.

The next section is devoted to one significant proof of the 1 -concavity property for data cost games.

\section{1-Concavity of the Data Cost Game}

Theorem 3. Every data cost game $\left\langle N, C_{\mathscr{D C}}\right\rangle$ of the form (1) satisfies 1-concavity.

Proof. Let $\left\langle N, C_{\mathscr{D} \mathscr{C}}\right\rangle$ be a data cost game. Fix coalition $S \subseteq N$, $S \neq N, S \neq \emptyset$. We establish the 1-concavity inequality (2) applied to $\left\langle N, C_{\mathscr{D} \mathscr{C}}\right\rangle$. Because of the compensation assumption $C_{\mathscr{D} \mathscr{C}}(N)=0$, the condition (2) reduces to

$$
\begin{gathered}
C_{\mathscr{D C}}(S) \geq \sum_{i \in N \backslash S} C_{\mathscr{D C}}(N \backslash\{i\}) \quad \text { or equivalently, by (1), } \\
c(D)-c\left(D_{S}\right) \geq \sum_{i \in N \backslash S}\left[c(D)-c\left(D_{N \backslash\{i\}}\right)\right]
\end{gathered}
$$

Write $N \backslash S=\left\{i_{1}, i_{2}, \ldots, i_{n-s}\right\}$ where $n-s$ denotes the cardinality of $N \backslash S$. Define, for every $0 \leq k \leq n-s$, the data set $A_{i_{k}}=D_{S} \bigcup_{\ell=1}^{k} D_{i_{\ell}}$, where $A_{i_{0}}=D_{S}, A_{i_{n-s}}=D_{N}=D$. In this setting, using a telescoping sum, (4) is equivalent to

$$
\sum_{k=1}^{n-s}\left[c\left(A_{i_{k}}\right)-c\left(A_{i_{k-1}}\right)\right] \geq \sum_{k=1}^{n-s}\left[c(D)-c\left(D_{N \backslash\left\{i_{k}\right\}}\right)\right] .
$$

In view of (5), it suffices to show the following: for all $1 \leq k \leq$ $n-s$

$$
c\left(A_{i_{k}}\right)-c\left(A_{i_{k-1}}\right) \geq c(D)-c\left(D_{N \backslash\left\{i_{k}\right\}}\right) \quad \text { or equivalently, }
$$

$$
\sum_{j \in A_{i_{k}} \backslash A_{i_{k-1}}} c_{j} \geq \sum_{j \in D \backslash D_{N \backslash\left\{i_{k}\right\}}} c_{j}
$$


In view of (7), in turn, it suffices to show the inclusion $D \backslash$ $D_{N \backslash\left\{i_{k}\right\}} \subseteq A_{i_{k}} \backslash A_{i_{k-1}}$ for all $1 \leq k \leq n-s$. Finally, note that $j \in$ $D \backslash D_{N \backslash\left\{i_{k}\right\}}$ means $j \in D_{i_{k}}$, but $j \notin D_{i_{\ell}}$ for all $\ell \neq k, \ell \in N$ yielding $j \notin D_{S}$ holds for any $S \not k$. Thus, $j \notin A_{i_{k-1}}$ and $j \in$ $A_{i_{k}}$.

Notice that the equivalence of (6) and (7) in the proof of Theorem 3 is due to the additive cost assumption in that $c(A)=\sum_{j \in A} c_{j}$ for any data subset $A \subseteq D$. We claim that the 1concavity property is still valid when the characteristic cost function $C_{\mathscr{D C}}: \mathscr{P}(N) \rightarrow \mathbb{R}$ is of the following generalized form: there exists a real number $\beta \in\{1,1 / 2,1 / 3, \ldots\}$ such that

$$
C_{\mathscr{C D}}(S)=\left[\sum_{j \in D} c_{j}\right]^{\beta}-\left[\sum_{j \in D_{S}} c_{j}\right]^{\beta} \quad \forall S \subseteq N, S \neq \emptyset .
$$

By (8), the data cost of coalition $S$ equals the surplus of costs of data that the coalition does not own; where the surplus is measured by some concave utility function $u(x)$ of the form $x^{1 / \alpha}$ such that $\alpha$ is any natural number (the case $\alpha=1$ agrees with the additive cost setting).

Theorem 4. Every generalized data cost game $\left\langle N, C_{\mathscr{D} \mathscr{C}}\right\rangle$ of the form (8) satisfies the 1-concavity property.

Proof. It suffices to prove the equivalent version of (6) as follows: for all $1 \leq k \leq n-s$

$$
\left[\sum_{j \in A_{i_{k}}} c_{j}\right]^{\beta}-\left[\sum_{j \in A_{i_{k-1}}} c_{j}\right]^{\beta} \geq\left[\sum_{j \in D} c_{j}\right]^{\beta}-\left[\sum_{j \in D_{N \backslash\left\{i_{k}\right\}}} c_{j}\right]^{\beta} .
$$

Write $\alpha=1 / \beta$. We make use of the fundamental calculus relationship:

$$
x-y=\left[x^{\beta}-y^{\beta}\right] \cdot\left[\sum_{p=0}^{\alpha-1}\left(x^{\beta}\right)^{\alpha-1-p} \cdot\left(y^{\beta}\right)^{p}\right] \quad \forall x, y \in \mathbb{R} .
$$

Fix $1 \leq k \leq n-s$. This fundamental calculus relationship applied to the validity of (6) yields

$$
\begin{gathered}
{\left[\left[\sum_{j \in A_{i_{k}}} c_{j}\right]^{\beta}-\left[\sum_{j \in A_{i_{k-1}}} c_{j}\right]^{\beta}\right] \cdot A} \\
\geq\left[\left[\sum_{j \in D} c_{j}\right]^{\beta}-\left[\sum_{\left.j \in D_{N \backslash\left\{i_{k}\right\}} c_{j}\right]}\right]^{\beta}\right] B,
\end{gathered}
$$

where the two real numbers $A$ and $B$ are given by

$$
\begin{aligned}
& A=\sum_{p=0}^{\alpha-1}\left[\sum_{j \in A_{i_{k}}} c_{j}\right]^{(\alpha-1-p) / \alpha} \cdot\left[\sum_{j \in A_{i_{k-1}}} c_{j}\right]^{p / \alpha}, \\
& B=\sum_{p=0}^{\alpha-1}\left[\sum_{j \in D} c_{j}\right]^{(\alpha-1-p) / \alpha} \cdot\left[\sum_{\left.j \in D_{N \backslash\left\{i_{k}\right\}} c_{j}\right]^{p / \alpha} .}\right.
\end{aligned}
$$

Note that $A \leq B$ due to the sum of increasing functions $x^{q}$, where $q>0$. From (11), together with $A \leq B$, we conclude that (9) holds.

Corollary 5. According to the theory developed for n-person 1-concave cost games $\langle N, C\rangle[5]$, the so-called nucleolus cost allocation $\vec{y}=\left(y_{i}\right)_{i \in N} \in \mathbb{R}^{N}$ for any data cost game $\left\langle N, C_{\mathscr{D} \mathscr{C}}\right\rangle$ is given by

$$
y_{i}=\Delta_{i}\left(N, C_{\mathscr{D} \mathscr{C}}\right)-\frac{1}{n} \cdot\left[\sum_{j \in N} \Delta_{j}\left(N, C_{\mathscr{D} \mathscr{C}}\right)-C_{\mathscr{D} \mathscr{C}}(N)\right] .
$$

Because $C_{\mathscr{D C}}(N)=0$, it holds $\Delta_{i}\left(N, C_{\mathscr{D C}}\right)=-C_{\mathscr{D C}}(N \backslash\{i\})$ for all $i \in N$ and so, (13) simplifies as follows: for all $i \in N$,

$$
\begin{aligned}
& y_{i}=-C_{\mathscr{D} \mathscr{C}}(N \backslash\{i\})+\frac{\Delta\left(N, C_{\mathscr{D} \mathscr{C}}\right)}{n}, \\
& \text { where } \Delta\left(N, C_{\mathscr{D} \mathscr{C}}\right)=\sum_{j \in N} C_{\mathscr{D} \mathscr{C}}(N \backslash\{i\}) .
\end{aligned}
$$

According to (14), a player $i$ receives a compensation which equals $C_{\mathscr{D C}}(N \backslash\{i\})$ and loses the average of the total coalitional cost the amount of which is $(1 / n) \cdot \sum_{j \in N} C_{\mathscr{D C}}(N \backslash\{i\})$. In particular, $y_{i}<0$ if and only if $C_{\mathscr{D C}}(N \backslash\{i\})>\Delta\left(N, C_{\mathscr{D C}}\right) / n$. In words, according to the nucleolus, a player $i$ receives a compensation if and only if the coalitional cost $C_{\mathscr{D C}}(N \backslash\{i\})$ strictly majorizes the average of such expressions; that is, the $(n-1)$ person coalition not containing player $i$ owns sufficiently few data.

\section{1-Concavity of Bicycle Cost Games}

Throughout this section write $N=\{1,2, \ldots, n\}$ and suppose that the individual data sets $D_{i} \subseteq D, i \in N$, are nested which fits particular situations like, for instance, joint ventures between firms whose $R+D$ programs are at different stages of progress [8].

Let us consider the decreasing sequence of individual data sets in that $D_{1} \supseteq D_{2} \supseteq \cdots \supseteq D_{n}$. Under these circumstances, the data cost game $\left\langle N, C_{\mathscr{D} \mathscr{C}}\right\rangle$ of the form (1) satisfies the increasing sequence $0=C_{\mathscr{D} \mathscr{C}}(\{1\}) \leq C_{\mathscr{D C}}(\{2\}) \leq \cdots \leq$ $C_{\mathscr{D C}}(\{n\})$, as well as $C_{\mathscr{D C}}(S)=0$ for all $S \subseteq N$ with $1 \in S$, in particular $C_{\mathscr{D} \mathscr{C}}(N \backslash\{i\})=0$ for all $i \in N \backslash\{1\}$, whereas $C_{\mathscr{D C}}(N \backslash\{1\})=c\left(D_{1} \backslash D_{2}\right)$. Additionally, this type of data cost game satisfies the following relationship (which remains valid in case of an increasing sequence of individual data sets):

$$
C_{\mathscr{D C}}(S)=\min _{i \in S} C_{\mathscr{D C}}(\{i\}) \quad \forall S \subseteq N, S \neq \emptyset .
$$

Write $S=\left\{i_{1}, i_{2}, \ldots, i_{s}\right\}$ such that $i_{1}<i_{2}<\cdots<i_{s}$. Because $D_{i_{1}} \supseteq D_{i_{2}} \supseteq \cdots \supseteq D_{i_{s}}$, it holds $C_{\mathscr{D C}}\left(\left\{i_{1}\right\}\right) \leq$ $C_{\mathscr{D C}}\left(\left\{i_{2}\right\}\right) \leq \cdots \leq C_{\mathscr{D C}}\left(\left\{i_{s}\right\}\right)$. Moreover, $D_{S}=D_{i_{1}}$, and therefore, $C_{\mathscr{D} \mathscr{C}}(S)=C_{\mathscr{D C}}\left(\left\{i_{1}\right\}\right)=\min _{i \in S} C_{\mathscr{D} \mathscr{C}}(\{i\})$. The purpose of the remainder of this section is to show that the 1concavity property remains valid for cost games $\langle N, C\rangle$ of the form (15) with arbitrary (not necessary zero) stand-alone costs $C(\{i\}), i \in N$. 
Definition 6. A cooperative cost game $\langle N, C\rangle$ with player set $N$ is called a bicycle cost game and an airport cost game [9] if its characteristic cost function $C: \mathscr{P}(N) \rightarrow \mathbb{R}$ satisfies

$$
\begin{array}{r}
C(S)=\min _{i \in S} C(\{i\}) \text { respectively } C(S)=\max _{i \in S} C(\{i\}) \\
\forall S \subseteq N, S \neq \emptyset .
\end{array}
$$

In the setting of owners of bicycles, any group of cyclists is not willing to spend more than the cheapest repairing cost of the best bicycle. In the setting of landings by different types of airplanes at some runway, the largest type needs the longest runway, yielding the highest stand-alone cost.

Theorem 7. Every bicycle cost game $\langle N, C\rangle$ of the form (16) satisfies 1-concavity.

Proof. Let $\langle N, C\rangle$ be a bicycle cost game. Without loss of generality, suppose that the stand-alone costs are ordered such that $0 \leq C(\{1\}) \leq C(\{2\}) \leq \cdots \leq C(\{n\})$. We establish the 1-concavity inequalities (2) and (3) applied to the bicycle cost game. Firstly, $C(N)=C(\{1\})$ and secondly, the marginal costs satisfy $\Delta_{i}(N, C)=C(N)-C(N \backslash\{i\})=0$ for all $i \in N \backslash\{1\}$, whereas $\Delta_{1}(N, C)=C(N)-C(N \backslash\{1\})=C(\{1\})-C(\{2\})$.

Fix coalition $S \subseteq N, S \neq \emptyset$. We distinguish two types of coalitions $S$. In case $1 \in S$, then $\Delta_{i}(N, C)=0$ for all $i \in N \backslash S$, whereas $C(S)=C(\{1\})=C(N)$ and, in turn, the 1-concavity condition (2) is met as a system of equalities. In case $1 \in N \backslash S$, then (2) reduces to $C(N) \leq C(S)+C(N)-C(N \backslash\{1\})$ or, equivalently, $C(S) \geq C(\{2\})$ and hence, the 1-concavity property holds too if $1 \notin S$. This proof technique illustrates that the largest stand-alone costs $C(\{k\}), 3 \leq k \leq n$, do not matter for the 1-concavity property as long as their truncation remains above the second smallest stand-alone cost $C(\{2\})$. In this setting, (3) holds trivially.

Corollary 8. According to the nucleolus cost allocation (13) applied to bicycle cost games, the second smallest stand-alone cost $C(\{2\})$ is charged equally to all players, except for the player with the smallest stand-alone cost who receives a compensation amounting the difference between both stand-alone costs. In formula, $\mu_{i}(N, C)=C(\{2\}) / n$ for all $i \in N \backslash\{1\}$ and $\mu_{1}(N, C)=$ $\mu_{2}(N, C)-[C(\{2\})-C(\{1\})]$.

The proposed new basis has been introduced and developed in [10] as a subclass of 1-concave $n$-person games, which are called complementary unanimity cost games.

Definition 9 (see [10] with adapted notation). With every coalition $T \subseteq N, T \neq N, T \neq \emptyset$, there is associated complementary unanimity cost game $\left\langle N, C_{T}\right\rangle$ given by

$$
C_{T}(S)= \begin{cases}1 & \text { if } S \neq \emptyset, S \cap T=\emptyset \\ 0 & \text { if } S=\emptyset \text { or } S \cap T \neq \emptyset\end{cases}
$$

In addition, the complimentary unanimity cost game $\left\langle N, C_{\emptyset}\right\rangle$ is given by $C_{\emptyset}(\emptyset)=0$ and $C_{\emptyset}(S)=1$ otherwise. Note that $C_{T}(N)=0$ for all $T \varsubsetneqq N$, except $T=\emptyset$.
Corollary 10. As shown in [10], the well-known Shapley cost allocation charged to the agents of anyn-person complementary unanimity cost game $\left\langle N, C_{T}\right\rangle$ amounts

$$
\begin{gathered}
\operatorname{Sh}_{i}\left(N, C_{T}\right)=\frac{1}{n} \quad \forall i \in N \backslash T, \\
\operatorname{Sh}_{i}\left(N, C_{T}\right)=\frac{1}{n}-\frac{1}{|T|} \quad \forall i \in T .
\end{gathered}
$$

Theorem 11. Suppose without loss of generality $0 \leq C(\{1\}) \leq$ $\cdots \leq C(\{n\})$. Every n-person bicycle cost game $\langle N, C\rangle$ can be decomposed as the following linear combination of a number of complementary unanimity cost games with nonnegative coefficients:

$$
\begin{array}{r}
C=\sum_{j=0}^{n-1}[C(\{j+1\})-C(\{j\})] \cdot C_{L_{j}}, \\
\text { where } L_{0}=\emptyset, L_{j}=\{1,2, \ldots, j\} \\
\forall j \in N .
\end{array}
$$

The Shapley cost allocation $\operatorname{Sh}(N, C)$ for an $n$-person bicycle cost game $\langle N, C\rangle$ equals

$$
S h_{i}(N, C)=-\sum_{j=i}^{n} \frac{C(\{j+1\})-C(\{j\})}{j}
$$

$\forall i \in N, \quad$ where $C(\{n+1\})=0$.

The Shapley cost allocation $\operatorname{Sh}(N, C)$ for an n-person airport cost game $\langle N, C\rangle$ equals

$$
S h_{i}(N, C)=\sum_{j=0}^{i-1} \frac{C(\{j+1\})-C(\{j\})}{n-j}
$$

$\forall i \in N$, where $C(\{0\})=0$.

Proof. Fix coalition $S \subseteq N, S \neq \emptyset$. Write $C(S)=C(\{k\})$ such that $k \in S$ and $\ell \notin S$ for all $1 \leq \ell<k$. Given any $0 \leq j \leq n-1$, the following equivalences hold: $C_{L_{j}}(S)=1$ if and only if $S \cap L_{j}=\emptyset$ if and only if $0 \leq j<k$. From this, we derive the validity of (19). The validity of (20) is left for the reader, applying the additivity property of the Shapley cost allocation to (19) and taking into account (18) as listed in Corollary 10.

Because of the relationship $\max _{i \in S} C(\{i\})=C(\{n\})-$ $\min _{i \in S}[C(\{n\})-C(\{i\})]$ for all $S \subseteq N, S \neq \emptyset$, every $n$-person airport cost game with stand-alone costs $C(\{i\}), i \in N$, ordered as an increasing sequence, is associated with a bicycle cost game with adapted stand-alone costs $C(\{n\})-C(\{i\}), i \in N$, to be ordered as an increasing sequence. In this setting, (21) is a direct consequence of (20) applied to this latter bicycle cost game.

Remark 12. It is left for the reader to check that the Shapley value of the form (20) can be written alternatively as follows:

$$
\operatorname{Sh}_{i}(N, C)=\frac{C(\{i\})}{i}-\sum_{k=i+1}^{n} \frac{C(\{k\})}{k \cdot(k-1)} \quad \forall i \in N \text {. }
$$


According to the Shapley value of Bicycle Cost Games, we can understand it as follows: for the ordered cyclists with $C(\{1\}) \leq C(\{2\}) \leq \cdots \leq C(\{n\})$, in the beginning, there is only one cyclist 1 ; the cost of the repairing fee for him is $C(\{1\})$; then player 2 is involved in which makes the cost of repairing fee of player 1 less and the decreasing amount equals $C(\{2\}) / 2$ while the cost of player 2 is $C(\{2\}) / 2$; after that, player 3 joins in which makes the cost of players 1 and 2 less and the total decreasing amount is $C(\{3\}) / 3$ which is divided equally between players 1 and 2 while the cost of player 2 is $C(\{3\}) / 3$;. . ; finally, player $n$ joins in; the cost of him equals $C(\{n\}) / n$, while this amount is divided equally among the other $n-1$ players.

\section{Concluding Remarks}

The proof of the 1-concavity property for data cost games is treated in Section 2. Section 3 establishes the 1-concavity property for a related class of games, called bicycle cost games. Due to 1-concavity, the formula (13) for the nucleolus cost allocation is fully determined in terms of the marginal costs $\Delta_{i}(N, C)=C(N)-C(N \backslash\{i\}), i \in N$, together with $C(N)=0$. Results about the core for both data cost games and bicycle cost games are beyond the scope of this paper and can be found in $[1,8]$. Finally, an alternative proof of the main Theorem 3 is treated in [11] in terms of Dutch soccer teams and their potential fans. Three other applications of oneconcavity or one-convexity, called library game, coinsurance game, and the dual game of the Stackelberg oligopoly game, respectively, can be found in $[10,12,13]$. The nucleolus for 2 convex games is treated in [14]. The search for other appealing classes of cost games satisfying the 1-concavity property is still going on.

\section{Conflict of Interests}

The authors declare that there is no conflict of interests regarding the publication of this paper.

\section{Acknowledgment}

The first author Dongshuang Hou acknowledges financial support by National Science Foundation of China (NSFC) through Grant nos. 71171163, 71271171, and 31300310.

\section{References}

[1] P. Dehez and D. Tellone, "Data games: sharing public goods with exclusion," Journal of Public Economic Theory, vol. 15, no. 4, pp. 654-673, 2013.

[2] A. E. Roth, The Shapley Value: Essays in Honor of Lloyd S. Shapley, Cambridge University Press, New York, NY, USA, 1988.

[3] L. S. Shapley, "A value for n-person games," in Annals of Mathematics Study, vol. 28, pp. 307-317, Princeton University Press, Princeton, NJ, USA, 1953.

[4] D. Schmeidler, "The nucleolus of a characteristic function game," SIAM Journal on Applied Mathematics, vol. 17, pp. 11631170, 1969.
[5] T. S. H. Driessen, Cooperative Games, Solutions, and Applications, Kluwer Academic Publishers, Dordrecht, The Netherlands, 1988.

[6] T. S. H. Driessen, "Properties of 1-convex n-person games," Operations-Research-Spektrum, vol. 7, no. 1, pp. 19-26, 1985.

[7] T. S. H. Driessen and S. H. Tijs, "The t-value, the nucleolus and the core for a subclass of games," Methods Operations Research, vol. 46, pp. 395-406, 1983.

[8] P. Dehez, "Cooperative provision of indivisible public goods," Theory and Decision, vol. 74, no. 1, pp. 13-29, 2013.

[9] S. C. Littlechild and G. Owen, "A simple expression for the Shapley value in a special case," Management Science, vol. 20, pp. 370-372, 1973.

[10] T. S. H. Driessen, A. B. Khmelnitskaya, and J. Sales, "1-concave basis for TU games and the library game," TOP, vol. 20, no. 3, pp. 578-591, 2012.

[11] D. Hou and T. Driessen, "Interaction between Dutch soccer teams and fans: a mathematical analysis through cooperative game theory," Applied Mathematics, vol. 3, no. 1, pp. 86-91, 2012.

[12] T. S. H. Driessen, V. Fragnelli, I. V. Katsev, and A. B. Khmelnitskaya, "On 1-convexity and nucleolus of co-insurance games," Insurance, vol. 48, no. 2, pp. 217-225, 2011.

[13] T. S. H. Driessen, D. Hou, and A. Lardon, "Stackelberg oligopoly TU-games: characterization of the core and 1-concavity of the dual game," Working Paper, University of St. Étienne, SaintÉtienne, France, 2012.

[14] T. S. H. Driessen and D. Hou, "A note on the nucleolus for 2convex TU games," International Journal of Game Theory, vol. 39, no. 1-2, pp. 185-189, 2010. 


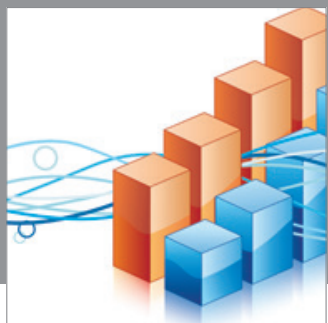

Advances in

Operations Research

mansans

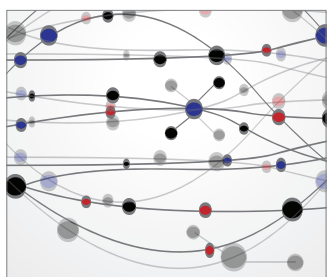

The Scientific World Journal
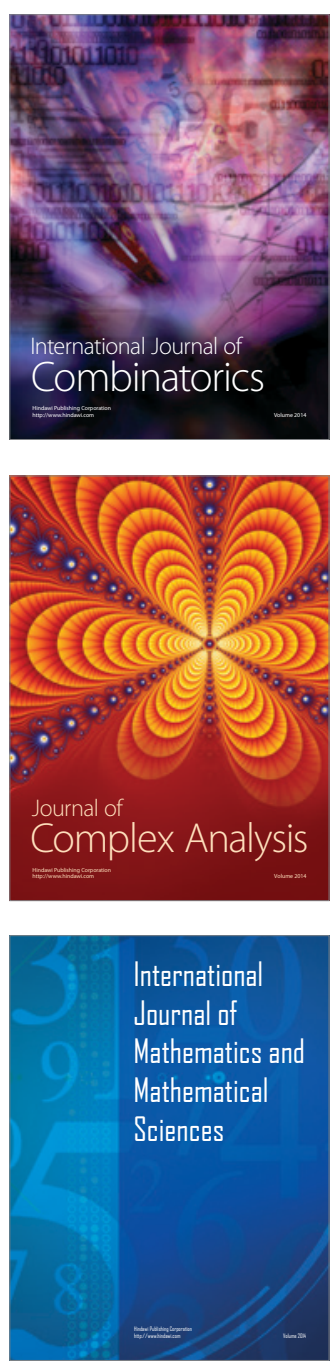
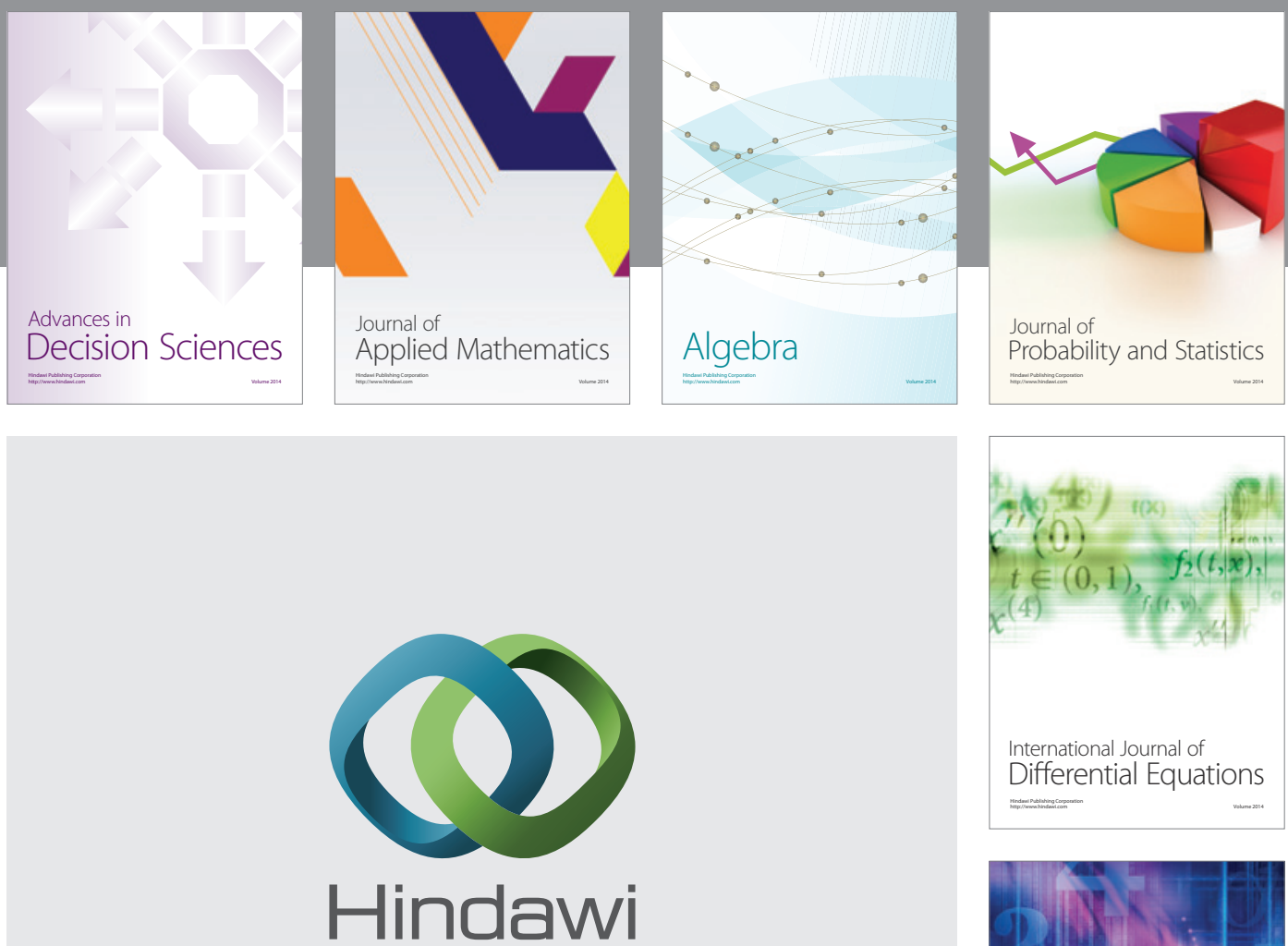

Submit your manuscripts at http://www.hindawi.com
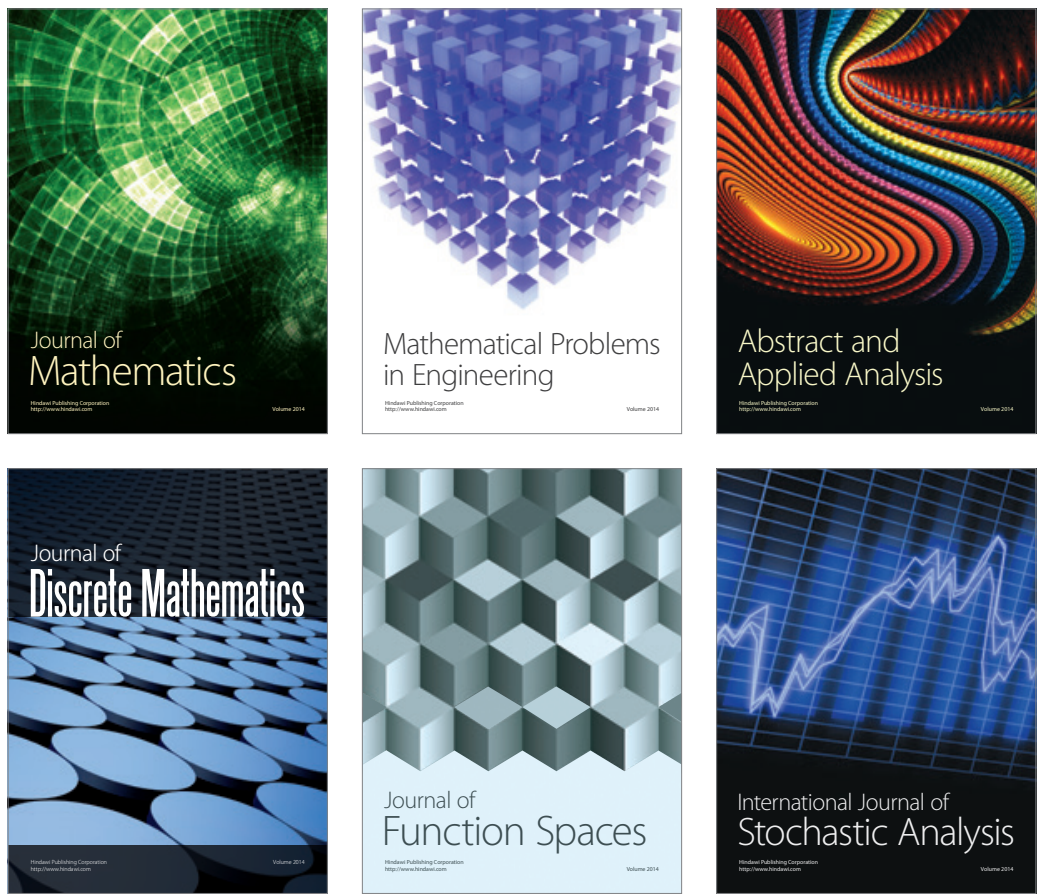

Journal of

Function Spaces

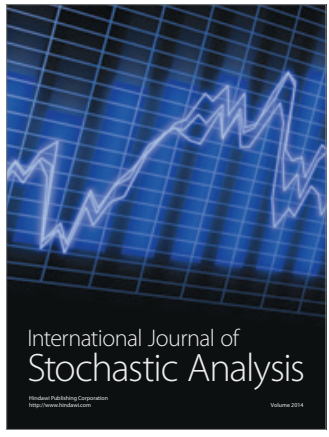

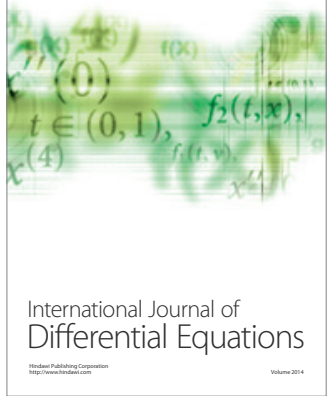
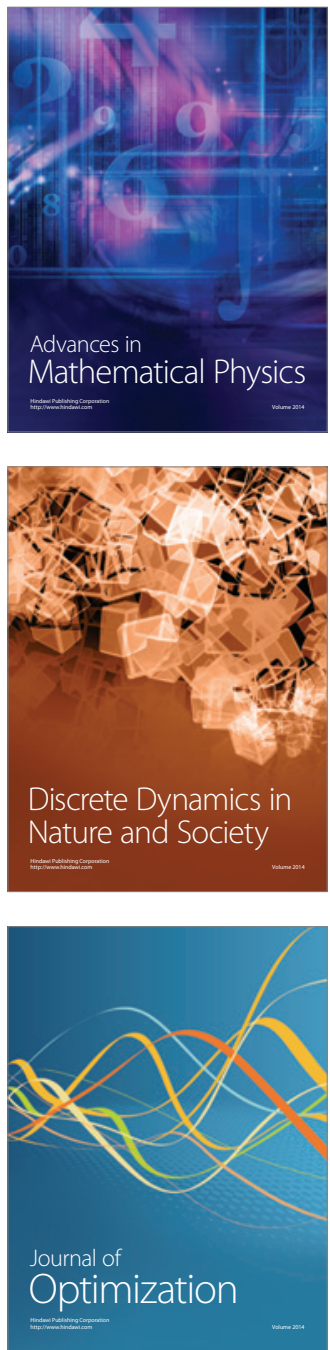